\title{
The day after tomorrow: cardiac surgery post-COVID-19.
}

\author{
Bessem Gara $\mathrm{Ali}^{1}$, Tarek Announe ${ }^{2}$, and Gilles Amr ${ }^{3}$ \\ ${ }^{1}$ Hôpital Bichat - Claude-Bernard \\ ${ }^{2}$ Centre Hospitalier Universitaire de Saint-Etienne \\ ${ }^{3}$ Centre Hospitalier Universitaire de Reims Pole Thoracique Cardiaque Vasculaire
}

June 22, 2020

\begin{abstract}
Humanity is experiencing the worst global disaster since the Second World War. In deed, Covid19 has affected more than 8,500,000 patients and more than 450,000 have died worldwide. Cardiac surgeons have been able to adapt to the situation by adopting new methods such as remote consultations and telemedicine. This brief communication summarizes the current situation and anticipates the resumption of the elective activity.
\end{abstract}

\section{Manuscript Text:}

To date on 20 June 2020, more than 8,500,000 patients have been affected by Covid19 and more than 450,000 have died worldwide (1). The epidemic that initially started in Hubei Province in China at the end of December 2019 has rapidly spread worldwide to 200 countries (2). The pandemic is improving according to the latest figures in the United States, the most affected country, and for the rest of the world except Latine America.

Our policy as cardiac surgeons throughout this period of lock-down was to delay all elective procedures on the one hand, and to refer patients to less invasive percutaneous coronary intervention (PCI), transcatheter aortic valve replacement (TAVR) or transcatheter mitral valve replacement (TMVR) procedures on the other hand. Most cardiac surgeons limited surgery to cases such as acute aortic dissections, emergency coronary artery syndromes not amenable to PCI or time-sensitive valve surgery not amenable to TAVR or TMVR (3). With the first signs of resolution of the pandemic and the start of delivery in several European countries, a significant number of patients are expected to be treated: patients who are symptomatic but who preferred not to consult during the lockdown period and asymptomatic ones who had delayed or cancelled appointments for follow-up of their chronic diseases. Elderly, overweight, hypertensive and diabetic male patients are currently known to pay the highest price and mortality in these patients is higher than in the rest of the population (4).

Given that elective cardiac surgery activity has fallen by at least $50 \%$ according to Salenger et al, the postCovid19 period should result in an increase of $216 \%$ to $263 \%$ per month in the number of patients to be treated (5). The period required to absorb the delay in patient management will vary between 1 and 8 months depending on the date of resumption of full activities and the capacity of hospitals to absorb these volumes (5). There will be coronary patients who have had an inadequately managed myocardial infarction, resulting in complications such as heart failure, myocardial aneurysm, and mitral insufficiency due to chordal or papillary muscle rupture and other mechanical complications. There will be patients suffering from severe aortic valve stenosis presenting syncope, severe dyspnea at rest or resting angina. There will also be patients with severe mitral valve insufficiency complicated by acute pulmonary edema. 
These complicated patients have a longer intensive care unit (ICU) stay, require more resources and have a higher morbidity and mortality.

In order to be able to absorb these potentially complicated patients who were poorly followed during the pandemic, hospitals have to make the necessary material and human resources available. Unfortunately, they usually suffer from budget restrictions and are under the influence of drastic cost-cutting programs, which explains the challenges to increase their capacities. As things progress the intensive care unit beds dedicated to Covid19 become available, elective cardiac surgery activity will increase crescendo.

Residents as well as fellows who have been surgically on standby and who have been actively involved in resuscitation care in Covid19 units throughout the pandemic period should resume their training and education programs.

Given that the coronavirus may not disappear in the immediate future, and that the scenario of a second wave is plausible, cardiac surgical practice must adapt to these circumstances with an emphasis on frequent hand washing, barrier procedures, social and physical distancing. All scheduled patients for elective surgery should be screened for Covid19 prior to surgery and all emergent surgical patients should be considered Covid-positive until proven otherwise. This will break the chain of contamination and preserve the medical and paramedical teams. Caregivers affected by the Coronavirus have been quarantined for at least two weeks and until symptoms improve. This absenteeism has inevitably reduced the human capacities of the cardiac surgery and post-cardiac surgery resuscitation departments. A growing number of learned societies have begun to issue recommendations for the post-Covid19 period, such as the Canadian Society of Cardiac Surgeons, which has projected a return to normal surgical activity in three phases beginning with urgent patients and those who are less likely to require prolonged ICU and hospital length stay. The third phase is an increase of $100 \%$ of the capacity with a return to normal outpatient services while continuing to prioritize those at greatest risk on the wait list (6). During all phases patients on the wait list for surgery should be contacted to determine their symptoms to facilitate prioritization. Programs are encouraged to adopt a mechanism by which patients who are having increased symptoms or who are not doing well can contact the program to receive additional screening while waiting. Finally, the Extracorporeal Life Support Organization (ELSO) provides guidance regarding ventricular assist device (VAD), cardiac transplantation and mechanical circulatory support (including extracorporeal membrane oxygenation or ECMO)(6).

\section{References:}

1. WHO COVID-19 Dashboard. https://covid19.who.int/. Accessed 20 June 2020.

2. Coronavirus tracked: the latest figures as the pandemic spreads (Free to read). Financial Times.https://www.ft.com/coronavirus-latest

3. Fudulu DP, Angelini GD. Cardiac surgery in the time of the coronavirus. J Card Surg. 2020;13.https://doi.org/10.1111/jocs. 14580

4. Fauci AS, Lane HC, Redfield RR. Covid-19-navigating the uncharted. N Engl J Med. 2020;382(13):1268-1269. https://doi.org/10.1056/ NEJMe2002387

5. Salenger R, Etchill EW, Ad N, Matthew T, Alejo D, Whitman G, Lawton JS, Lau CL, Gammie CF, Gammie JS, The Surge after the Surge: Cardiac Surgery post-COVID- 19, The Annals of Thoracic Surgery (2020), doi: https://doi.org/10.1016/j.athoracsur.2020.04.018.

6. Hassan A, Arora RC, Lother SA, Adams C, Bouchard D, Cook R, GunningD, Lamarche Y, Malas T, Moon M, Ouzounian M, Rao V, Rubens F, Tremblay P, Whitlock R, Moss E, Légaré J-F, on behalf of the Canadian Society of Cardiac Surgeons, Ramping Up the Delivery of Cardiac Surgery During the COVID-19 Pandemic: A Guidance Statement from the Canadian Society of Cardiac Surgeons, Canadian Journal of Cardiology (2020), doi: https://doi.org/10.1016/j.cjca.2020.04.030. 